\title{
Palladium-catalyzed synthesis of novel tetra- and penta-cyclic biologically active benzopyran- and pyridopyran-containing heterocyclic systems
}

\author{
Tatjana Beresneva, Anatoly Mishnev, Elina Jaschenko, Irina Shestakova, \\ Anita Gulbe, and Edgars Abele*
}

Latvian Institute of Organic Synthesis, 21 Aizkraukles Street, Riga, LV-1006, Latvia

E-mail: $\underline{\text { abele@osi.lv }}$

\begin{abstract}
Syntheses of novel tetra- and penta-cyclic benzopyran and pyridopyran derivatives, via direct intramolecular arylation of 2-iodophenoxymethylhetarenes and 3-(2-bromo-pyridin-3yloxymethyl)-benzo[4,5]imidazo[2,1-b]thiazole in the catalytic system $\mathrm{Pd}(\mathrm{OAc})_{2} / \mathrm{Xantphos} \mathrm{/}$ $\mathrm{Cs}_{2} \mathrm{CO}_{3} / \mathrm{Ag}_{2} \mathrm{CO}_{3}$ in toluene, and a one-pot bicatalytic method for $12 \mathrm{H}$ [1]benzopyrano[3',4':4,5]thiazolo[3,2-a]benzimidazole directly from 3-chloromethylbenzo[4,5]imidazo[2,1- $b$ ]thiazole and 2-iodophenol, are described. This latter compound exhibits high cytotoxicity (MG-22A, $6 \mu \mathrm{g} / \mathrm{mL}$ ) on the mouse hepatoma cancer cell line and low toxicity $\left(\mathrm{LD}_{50}, 1058 \mathrm{mg} / \mathrm{kg}\right.$ ) on the mouse Swiss albino embryo fibroblasts 3T3.
\end{abstract}

Keywords: Palladium catalysis, intramolecular arylation, phase transfer catalysis, fused benzothiazoles, imidazoles, benzopyrans, pyridopyrans, cytotoxicity

\section{Introduction}

Pyrans and their benzo derivatives are of interest as biologically active compounds. ${ }^{1-3}$ The synthesis and reactions of pyrans and benzopyrans have been well reviewed..$^{4-9}$ Recently the uses and properties of important natural and synthetic $2 \mathrm{H}$-pyran-2-ones in organic synthesis were documented. $^{8,9}$ One of the earliest works describing the synthesis of the pyranothiazole ring from 3,5-dibromopyran-4-one and a thioamide was published in $1948 .{ }^{10}$ Some methods for the preparation of 2-substituted [1]benzopyrano[3,4- $d$ ]-thiazol-4-ones and -imidazol-4-ones are documented. ${ }^{11}$ More recently, the palladium-catalyzed synthesis of thiazolobenzopyran-2-ones from methyl 5-(2-allyloxyphenyl)thiazole-4-carboxylates was reported. ${ }^{12}$ Benzopyranothiazole and benzopyranothiophene ring systems were prepared by thermal intramolecular 1,3-dipolar cycloaddition of 2-(prop-2-ynyloxy- and cyanomethyloxy)-3,5-diphenyl-4-hydroxythiazolium hydroxides. ${ }^{13}$ Benzopyranoimidazoles have been prepared by electrochemical reduction / rearr- 
angement of benzopyranotriazines ${ }^{14}$ or by condensation of hydrazine with 4-oxochroman-3carbaldehyde $^{15}$.

An important modern development in the palladium-catalyzed synthesis of heterocyclic compounds using an intramolecular Heck-type reaction was recently highlighted in some reviews. ${ }^{16-19}$ Regioselective functionalization of the imidazole ring by transition metal-catalyzed $\mathrm{C}-\mathrm{N}$ and $\mathrm{C}-\mathrm{C}$ bond formation was reported in details by Rossi et al. ${ }^{20}$ Interestingly, that the pyrano[3',4':4,5]imidazo[1,2-a]pyridin-1-one ring system has been constructed by $\mathrm{Cu}(\mathrm{I})$ catalyzed cross-coupling and heterocyclization reactions of halogenated imidazopyridinecarboxylic acids in the presence of terminal alkynes. ${ }^{21}$

Our interest in polycyclic compounds containing imidazothiazole and related fragments was prompted by the wide range of biological activity of these heterocyclic systems. ${ }^{22-24}$ Furthermore, the synthesis of tetra- and penta-cyclic imidazolo- and thiazolo-benzopyran derivatives has not been investigated until now and so is the main aim of the present work. Beside this, selected stable compounds (4a, 10 and $\mathbf{1 3})$ were tested as cytotoxic agents.

\section{Results and Discussion}

Synthesis of the novel polycyclic compounds $\mathbf{4 a , b}, \mathbf{7 ,} 10$ and 13 was carried out in two steps. Alkylation of 2-iodophenol (2a) or 2-bromo-3-hydroxypyridine (2b) with chloromethylhetarenes $\mathbf{1}, \mathbf{5}, \mathbf{8}, \mathbf{1 1}^{24}$ was successfully achieved under phase transfer catalytic conditions - solid $\mathrm{KOH} /$ 18-crown-6 / toluene. Intermediates 3a,b, 6, 9 and 12 were isolated in 21-87\% yields. (Schemes $1-4)$

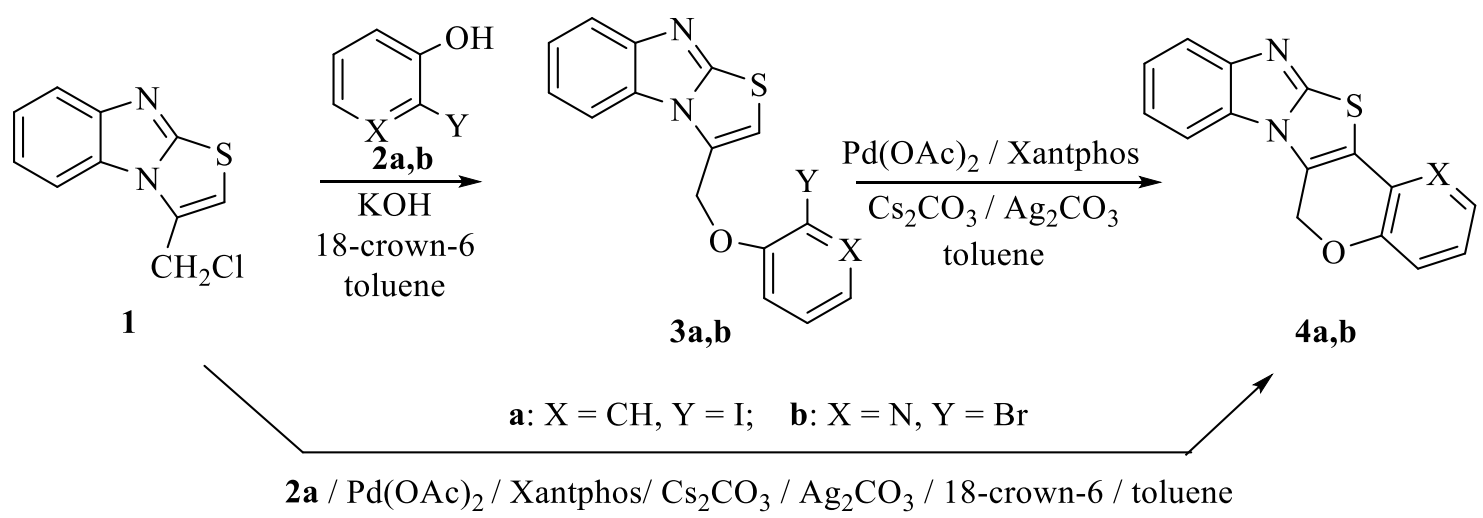

\section{Scheme 1}

The influence of catalyst, ligand and additive on the intramolecular Heck-type cyclization was studied in detail. Initially, we examined direct intramolecular arylation of 2-iodophenoxymethylhetarene 3a using $10 \mathrm{~mol} . \% \mathrm{Pd}\left(\mathrm{PPh}_{3}\right)_{4}$ as catalyst and 2 eq. $\mathrm{Cs}_{2} \mathrm{CO}_{3}$ as base. No product was found under these conditions. The use of the system $10 \mathrm{~mol} \% \mathrm{Pd}(\mathrm{OAc})_{2} / 20 \mathrm{~mol} \%$ 
Xantphos / $\mathrm{Cs}_{2} \mathrm{CO}_{3}$ (2 eq.) / toluene provided the polycycle 4a in a low (17\%) yield with 55\% conversion. The high activity of $\mathrm{Ag}_{2} \mathrm{CO}_{3}$ as an additive in Rh-catalyzed arylation of hetarenes was recently demonstrated. ${ }^{25}$ The addition of 0.5 eq. $\mathrm{Ag}_{2} \mathrm{CO}_{3}$ to the system $\mathrm{Pd}(\mathrm{OAc})_{2}(10 \mathrm{~mol} . \%)$ / Xantphos (20 mol.\%) / $\mathrm{Cs}_{2} \mathrm{CO}_{3}$ (2 eq.) / toluene furnished the desired product 4a in improved $(69 \%)$ yield with full conversion of the starting material 3a. All catalytic systems in the presence of $\mathrm{Ag}_{2} \mathrm{CO}_{3}$ were more active.

The polycycle 4a was also obtained directly from 3-chloromethylbenzo[4,5]imidazo[2,1b]thiazole (1) and 2-iodophenol (2a) in a one-pot synthesis using the bicatalytic system $\mathrm{Pd}(\mathrm{OAc})_{2}(10 \mathrm{~mol} . \%) / \mathrm{Xantphos}(20 \mathrm{~mol} . \%) / \mathrm{Cs}_{2} \mathrm{CO}_{3}$ (3 eq.) / $\mathrm{Ag}_{2} \mathrm{CO}_{3}$ (0,5 eq.) / 18-crown-6 (10 mol.\%), but in lower yield (25\%) than the two step synthesis provided.

The catalytic system $\mathrm{Pd}(\mathrm{OAc})_{2}\left(10\right.$ mol.\%) / Xantphos (20 mol.\%) / $\mathrm{Cs}_{2} \mathrm{CO}_{3}$ (2 eq. $) / \mathrm{Ag}_{2} \mathrm{CO}_{3}$ ( 0,5 eq. $) /$ toluene, as the most active, was used for the preparation of the polycyclic compounds 4b (Scheme 1), 7 (Scheme 2), 10 (Scheme 3) and 13 (Scheme 4). The products 4a,b, 7, 10 and 13 were isolated by column chromatography in $31-63 \%$ yields.

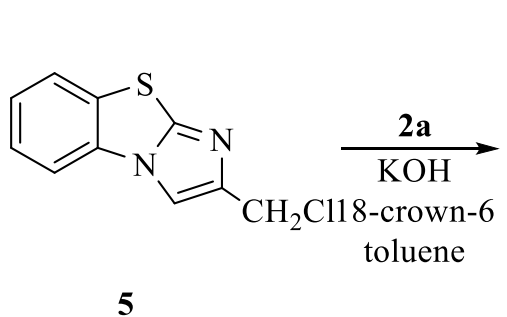

5<smiles>Ic1ccccc1OCc1cn2c(n1)sc1ccccc12</smiles>

$\underset{\mathrm{Cs}_{2} \mathrm{CO}_{3} / \mathrm{Ag}_{2} \mathrm{CO}_{3}}{\stackrel{\mathrm{Pd}(\mathrm{OAc})_{2} / \text { Xantphos }}{\longrightarrow}}$ toluene<smiles>c1ccc2c(c1)OCc1nc3sc4ccccc4n3c1-2</smiles>

7

Scheme 2

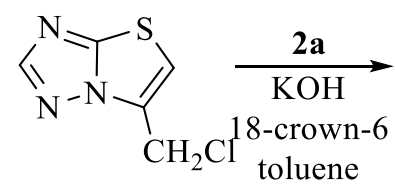

8

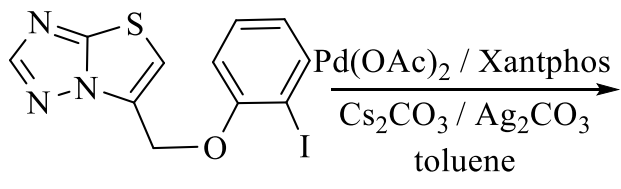

9

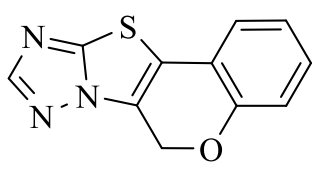

10

\section{Scheme 3}

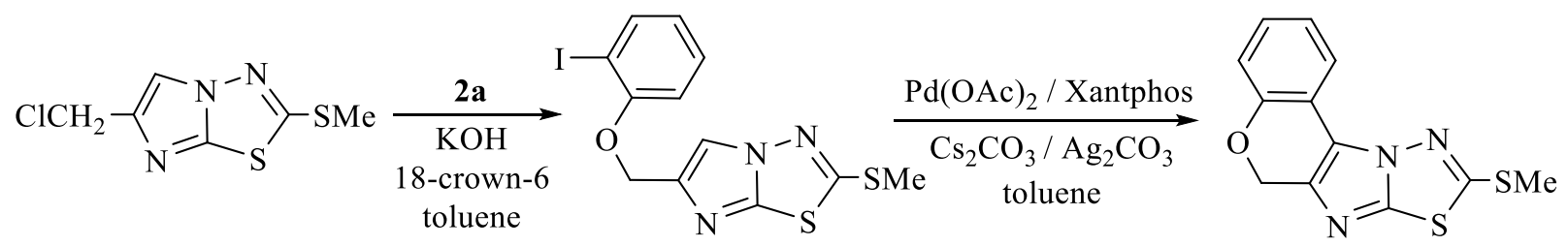

11

12

13

\section{Scheme 4}


The structure of compound $\mathbf{1 3}$ was confirmed by X-ray structural data. Needle like crystals of compound 13 suitable for intensity measurement were grown from chloroform. The entire molecule of polycycle $\mathbf{1 3}$ is essentially planar (Figure 1). The maximal deviation from the least squares mean plane drawn through all non-hydrogen atoms of the molecule is $0.398(2) \AA$ for the $\mathrm{O} 5$ atom. The molecule contains four condensed rings, the 1,3,4-thiadiazole (A), imidazole (B),

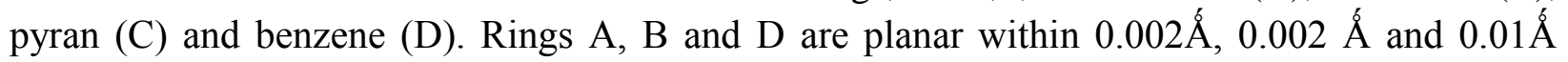
respectively. The pyran ring $(\mathrm{C})$ adopts a twist-half-chair conformation where atoms C6, C6a, $\mathrm{C} 10 \mathrm{~b}$ and $\mathrm{C} 10 \mathrm{c}$ form a strict plane $( \pm 0.005 \AA$ ) , while the $\mathrm{O} 5$ and $\mathrm{C} 4 \mathrm{a}$ atoms deviate from this plane by $0.564(4) \AA ̊$ and $0.258(4) \AA ̊$ respectively. The thiadiazolo-imidazole system (A+B) forms a dihedral angle of $9.5(1)^{\circ}$ with the benzene ring (D).

Bond lengths in compound $\mathbf{1 3}$ are in good agreement with the crystal structure of methyl 2-chloro-8-oxo-6H,8H-[1] benzopyrano[4',3':4,5]imidazo[2,1-b][1,3] thiazine-10-carboxylate ${ }^{26}$ having three similar condensed rings $(\mathrm{B}+\mathrm{C}+\mathrm{D}$ fragment), and of 6-(4-chlorophenyl)imidazo$[2,1-b][1,3,4]$ thiadiazole-2-sulfonamide ${ }^{27}$ with a similar thiadiazolo-imidazole system $(A+B)$. Intermolecular contacts are all of the van der Waals type.

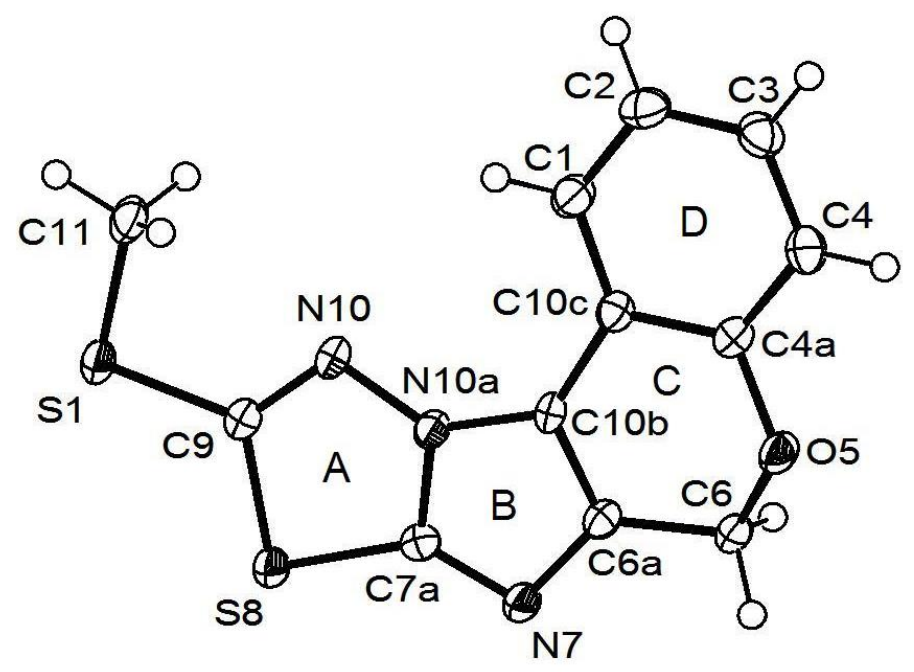

Figure 1. View of the molecule 13 with atomic numbering and ring labels. 
Table 1. Crystal data of the compound $\mathbf{1 3}$

\begin{tabular}{|c|c|}
\hline Empirical formula & $\mathrm{C}_{12} \mathrm{H}_{9} \mathrm{~N}_{3} \mathrm{OS}_{2}$ \\
\hline Formula weight & 275.34 \\
\hline Temperature $(\mathrm{K})$ & $180(2)$ \\
\hline Crystal system & orthorombic \\
\hline Space group & $P 2{ }_{1}{ }_{1} 2_{1}$ \\
\hline$a(\AA)$ & $3.9926(1)$ \\
\hline$b(\AA)$ & $10.2615(3)$ \\
\hline$c(\AA)$ & $27.847(1)$ \\
\hline Volume $\left(\AA^{3}\right)$ & $1140.88(6)$ \\
\hline Z & 4 \\
\hline Calc. density (Mg/m-3) & 1.603 \\
\hline Crystal size $(\mathrm{mm})=$ & $0.4 \times \square 0.1 \times \square 0.1$ \\
\hline Crystal colour & Colorless \\
\hline$\theta \square$ range $\left(^{\circ}\right)$ & $2.12-27.10$ \\
\hline \multirow[t]{3}{*}{ Index ranges } & $h-5 \rightarrow \square 5$ \\
\hline & $k-12 \rightarrow \square 13$ \\
\hline & $l-34 \rightarrow \square 35$ \\
\hline Data / Restraints / Parameters & $2279 / 0 / 163$ \\
\hline Final $R$ indices $[\mathrm{I}>2 \sigma \square(\mathrm{I})]$ & $0.0326 / 0.0670$ \\
\hline$R$ indices (all data) & $0.0417 / 0.0707$ \\
\hline Hydrogen atoms treatment & Constrained \\
\hline Largest diff. peak and hole (e. $\AA$-3) & 0.274 and -0.248 \\
\hline
\end{tabular}

Table 2. Cytotoxicity of polycyclic compounds $\mathbf{4 a}, \mathbf{1 0}$ and $\mathbf{1 3} \mathrm{IC}_{50}(\mu \mathrm{g} / \mathrm{mL})$

\begin{tabular}{cccc}
\hline Compound & HT-1080, $\mathrm{IC}_{50}$ & MG-22A, $\mathrm{IC}_{50}$ & $3 \mathrm{~T} 3, \mathrm{LD} 50, \mathrm{mg} / \mathrm{kg}$ \\
\hline $\mathbf{4 a}$ & 40 & 6 & 1058 \\
$\mathbf{1 0}$ & 20 & 28 & 1100 \\
$\mathbf{1 3}$ & 50 & 78 & 1487 \\
\hline
\end{tabular}

Cytotoxic activity of compounds 19 and 23 was tested in vitro on two monolayer tumor cell lines: MG-22A and HT-1080 (Table 2). Compound 4a exhibit high activity on the mouse hepatoma (MG-22A, $6 \mu \mathrm{g} / \mathrm{mL}$ ) cancer cell line. However, on the human fibrosarcoma cell line this compound is essentially inactive. Polycyclic compound $\mathbf{1 0}$ exhibit middle activity on both cancer cell lines. Compound $\mathbf{1 3}$ is inactive on the MG-22A and HT-1080 cancer cell lines.

Interestingly, that toxicity of compounds $\mathbf{4 a}, 10$ and 13 (LD $10,1058-1487 \mathrm{mg} / \mathrm{kg})$ detected on the mouse normal fibroblasts is not high. 


\section{Conclusions}

In summary, we have developed a facile method for synthesis of novel imidazole and thiazole containing benzopyran and pyridopyran derivatives via intramolecular cyclization of corresponding 2-iodophenoxy(or bromopyridin-3-yloxy)methylhetarenes in the catalytic system $\mathrm{Pd}(\mathrm{OAc})_{2} / \mathrm{Xantphos} / \mathrm{Cs}_{2} \mathrm{CO}_{3} / \mathrm{Ag}_{2} \mathrm{CO}_{3} /$ toluene. 12H-[1]Benzopyrano[3',4':4,5] thiazolo[3,2a]benzimidazole (4a) exhibit high cytotoxicity on the mouse hepatoma (MG-22A, $6 \mu \mathrm{g} / \mathrm{mL})$ cancer cell line and low toxicity on mouse Swiss Albino embryo fibroblasts (3T3, LD 501058 $\mathrm{mg} / \mathrm{kg})$.

\section{Experimental Section}

General. ${ }^{1} \mathrm{H}$ and ${ }^{13} \mathrm{C}$ NMR spectra were recorded on a Varian Mercury BB $400 \mathrm{MHz}$ in $\mathrm{CDCl}_{3}$ using HMDSO as internal standard. LC-MS spectra were recorded on Alliance Waters 2695 instrument and Waters 3100 mass detector. Column chromatography was performed with silica gel 0,035-0,070 nm (Acros). X-Ray diffraction data was collected using Nonius KappaCCD single crystal diffractometer (Bruker AXS) $\left(\mathrm{MoK} \alpha_{1}\right.$ - radiation, graphite monochromator). The structure was solved by SIR2004 ${ }^{28}$ and refined by SHELXL97 ${ }^{29}$ programs. Rms deviation of fitted atoms $=0.0066$. 3-Chloromethylbenzo[4,5]imidazo[2,1-b]thiazole (1), 2chloromethylbenzo[d]imidazo[2,1-b]thiazole (5), 6-chloromethylthiazolo[3,2-b][1,2,4]triazole (8), 6-chloromethyl-2-methylsulfanylimidazo[2,1-b][1,3,4]thiadiazole (11) were obtained by the procedure described in article. ${ }^{24}$ All prepared compounds are new and were characterized by melting point, LC-MS, HRMS, ${ }^{1} \mathrm{H}$ NMR and ${ }^{13} \mathrm{C}$ NMR spectra.

General procedure for synthesis of 2-iodophenoxymethylhetarenes 3a, 6, 9, 12 and 3-(2bromopyridin-3-yloxymethyl)-benzo[4,5]imidazo[2,1-b]thiazole (3b)

Solid pulverized $\mathrm{KOH}(0.98 \mathrm{~g}, 4.5 \mathrm{mmol})$ was added to solution of chloromethyl derivatives $\mathbf{1}, \mathbf{5}$, $\mathbf{8}$ or 11 (4 mmol), 2-iodophenol (2a) (0.88 g, 4 mmol) or 2-bromo-3-hydroxypyridine (2b) (0.87 $\mathrm{g}, 4 \mathrm{mmol})$, 18-crown-6 (0.1 g, $0.4 \mathrm{mmol})$ in toluene $(20 \mathrm{~mL})$. Reaction mixture was refluxed for $2 \mathrm{~h}$, cooled to room temperature, filtered and solvent was removed under reduced pressure. The products were purified using flash chromatography (silica, ethyl acetate). Spectroscopic characteristics.

3-(2-Iodophenoxymethyl)-benzo[4,5]imidazo[2,1-b]thiazole (3a). 66\% yield; mp 165-166 ${ }^{\circ} \mathrm{C}$; LC-MS, $407\left(\mathrm{M}^{+}+1\right)$; ${ }^{1} \mathrm{H}$ NMR $\delta(\square \mathrm{ppm} \square): 5.40$ (s, 2H, $\left.\mathrm{CH}_{2}\right), 6.82$ (t, 1H, J = $7.6 \mathrm{~Hz}, 4$ '-H), 6.86 (s, 1H, 2-H), 6.96 (d, 1H, J=8.4 Hz, 6'-H), 7.26 (t, 1H, J=8.0 Hz, 5'-H), 7.32-7.41 (m, $2 \mathrm{H}, 6-\mathrm{H}$ and 7-H), 7.80-7.84 (m, 3H, 5-H, 8-H and 3'-H); ${ }^{13} \mathrm{C} \mathrm{NMR} \delta$ ( $\square$ ppm $\left.\square\right): 63.80\left(\mathrm{CH}_{2}\right)$, $86.97,109.97,111.95,113.15,119.17,121.19$, 123.61, 124.15, 128.48, 129.55, 129.78, 140.09, 148.40, 156.20, 156.81 . 
3-(2-Bromopyridin-3-yloxymethyl)-benzo[4,5]imidazo[2,1-b]thiazole (3b). 44\% yield; mp 204-205 ${ }^{\circ} \mathrm{C}$; LC-MS, $361\left(\mathrm{M}^{+}+1\right)$; ${ }^{1} \mathrm{H}$ NMR $\delta$ ( $\square$ ppm $\square$ ): 5.75 (s, 2H, $\mathrm{CH}_{2}$ ), 7.26 and 7.35 (both t, $2 \mathrm{H}, J=7 \mathrm{~Hz}, 6-\mathrm{H}$ and 7-H), 7.50 (m, 1H, 5'-H), 7.52 (s, 1H, 2-H), 7.70 (d, 1H, $J=8 \mathrm{~Hz}, 4^{\prime}-$ $\mathrm{H}), 7.85-7.90(\mathrm{~m}, 2 \mathrm{H}, 5-\mathrm{H}$ and $8-\mathrm{H}), 8.05\left(\mathrm{~d}, 1 \mathrm{H}, J=5 \mathrm{~Hz}, 6-\mathrm{H}^{\prime}\right) ;{ }^{13} \mathrm{C}$ NMR $\delta(\square \mathrm{ppm} \square): 62.57$ $\left(\mathrm{CH}_{2}\right), 112.50,112.60,118.38,120.73,121.86,123.27,124.28,127.94,129.41,131.72,142.22$, $147.77,150.59,156.29$.

2-(2-Iodophenoxymethyl)-benzo[d]imidazo[2,1-b]thiazole (6). $21 \%$ yield; mp 175-177 ${ }^{\circ} \mathrm{C}$; LC-MS, $407\left(\mathrm{M}^{+}+1\right)$; ${ }^{1} \mathrm{H}$ NMR $\delta(\square \mathrm{ppm} \square): 5.27$ (s, 2H, $\left.\mathrm{CH}_{2}\right), 6.74$ (t, 1H, J = $7.2 \mathrm{~Hz}, 4$ '-H), $7.03\left(\mathrm{~d}, 1 \mathrm{H}, J=8.4 \mathrm{~Hz}, 6{ }^{\prime}-\mathrm{H}\right), 7.26-7.46$ (m, 3H, 5'-H, 6-H and 7-H), 7.60, 7.69 and 7.79 (all d, $3 \mathrm{H}, J=8.0 \mathrm{~Hz}, 3$ '-H, 5-H, 8-H), 7.85 (s, 1H, 3-H); ${ }^{13} \mathrm{C}$ NMR $\delta$ ( $\square$ ppm $\left.\square\right): 66.37\left(\mathrm{CH}_{2}\right), 86.73$, $110.07,112.80,112.97,115.19,122.94,124.33,124.91,126.14,129.53,138.40,139.45,143.99$, $147.47,157.11$.

6-(2-Iodophenoxymethyl)-thiazolo[3,2-b][1,2,4]triazole (9). 87\% yield; mp 121-122 ${ }^{\circ} \mathrm{C}$; LCMS, $358\left(\mathrm{M}^{+}+1\right)$; ${ }^{1} \mathrm{H}$ NMR $\delta(\square \mathrm{ppm} \square): 5.38\left(\mathrm{~s}, 2 \mathrm{H}, \mathrm{CH}_{2}\right), 6.80\left(\mathrm{t}, 1 \mathrm{H}, J=8.0 \mathrm{~Hz}, 4^{\prime}-\mathrm{H}\right), 6.96$ $(\mathrm{d}, 1 \mathrm{H}, J=8.4 \mathrm{~Hz}, 6$ '-H), $7.24(\mathrm{~d}, 1 \mathrm{H}, J=1.6 \mathrm{~Hz}, 5-\mathrm{H}), 7.34$ (t, 1H, $J=8.0 \mathrm{~Hz}, 5$ '-H), 7.82 (d, $1 \mathrm{H}, J=8.0 \mathrm{~Hz}, 3$ '-H), 8.19 (d, 1H, $J=1.2 \mathrm{~Hz}, 2-\mathrm{H}) ;{ }^{13} \mathrm{C}$ NMR $\delta$ ( $\left.\square \mathrm{ppm} \square\right): 63.39\left(\mathrm{CH}_{2}\right), 86.67$, $111.40,112.87,123.97,128.48,129.72,139.76,156.30,156.35,156.84$.

6-(2-Iodophenoxymethyl)-2-methylsulfanylimidazo[2,1-b][1,3,4]thiadiazole (12). 42\% yield; mp 124-125 ${ }^{\circ} \mathrm{C}$; LC-MS, $404\left(\mathrm{M}^{+}+1\right)$; ${ }^{1} \mathrm{H}$ NMR $\delta$ ( $\square$ ppm $\square$ ): 2.73 (s, 3H, CH 3 ), 5.19 (s, 2H, $\mathrm{CH}_{2}$ ), 6.72 (t, 1H, $\left.J=8.8 \mathrm{~Hz}, 5^{\prime}-\mathrm{H}\right), 6.99$ (d, 1H, $J=8.4 \mathrm{~Hz}, 6$ '-H), 7.29 (t, 1H, J=8.4 Hz, 4'H) $7.78\left(\mathrm{~d}, 1 \mathrm{H}, J=7.6 \mathrm{~Hz}, 3\right.$ '-H), $7.83(\mathrm{~s}, 1 \mathrm{H}, 5-\mathrm{H}) ;{ }^{13} \mathrm{C}$ NMR $\delta(\square \mathrm{ppm} \square): 16.07(\mathrm{Me}), 66.80$ $\left(\mathrm{CH}_{2}\right), 86.80,112.54,112.95,122.82,129.44,139.46,142.00,144.21,157.06,161.44$.

General procedure for synthesis of polycyclic compounds 4a,b, 7, 10 and 13

Mixture of 2-iodophenoxymethylhetarenes 3a, 6, 9, 12 or 3-(2-bromo-pyridin-3-yloxymethyl)benzo[4,5]imidazo[2,1- $b$ ] thiazole $3 \mathbf{b}(0.49 \mathrm{mmol}), \mathrm{Pd}(\mathrm{OAc})_{2}(0.011 \mathrm{~g}, 0.049 \mathrm{mmol})$, Xantphos (0.057 g, $0.098 \mathrm{mmol}$ ), anhydrous $\mathrm{Cs}_{2} \mathrm{CO}_{3}(0.32 \mathrm{~g}, 0.98 \mathrm{mmol})$ and $\mathrm{Ag}_{2} \mathrm{CO}_{3}(0.068 \mathrm{~g}, 0.25 \mathrm{mmol})$ in dry toluene $(10 \mathrm{~mL})$ was heated at $120^{\circ} \mathrm{C}$ for $24 \mathrm{~h}$ in glass reactor under argon. Reaction mixture was filtered and solvent was removed under reduced pressure. The products were purified using flash chromatography (silica, ethyl acetate : hexane (1:1)). Spectroscopic characteristics:

$12 H$-[1]Benzopyrano $\left[3^{\prime}, 4^{\prime}: 4,5\right]$ thiazolo[3,2-a]benzimidazole (4a). 69\% yield; $\mathrm{mp}>230{ }^{\circ} \mathrm{C}$; LC-MS, $279\left(\mathrm{M}^{+}+1\right) ;{ }^{1} \mathrm{H}$ NMR $\delta(\square \mathrm{ppm} \square): 5.80$ (s, 2H, $\left.\mathrm{CH}_{2}\right), 6.96$ (d, $\left.1 \mathrm{H}, J=8.0 \mathrm{~Hz}, 4-\mathrm{H}\right)$, $7.02(\mathrm{t}, 1 \mathrm{H}, J=8.0 \mathrm{~Hz}, 2-\mathrm{H}), 7.10(\mathrm{~d}, 1 \mathrm{H}, J=7.6 \mathrm{~Hz}, 1-\mathrm{H}), 7.18(\mathrm{t}, 1 \mathrm{H}, J=7.6 \mathrm{~Hz}, 3-\mathrm{H}), 7.26$ and 7.36 (both t, $2 \mathrm{H}, J=8.0 \mathrm{~Hz}, 8-\mathrm{H}$ and 9-H), 7.53 and 7.79 (both d, 2H, $J=8.0 \mathrm{~Hz}, 7-\mathrm{H}$ and $10 \mathrm{H}) ;{ }^{13} \mathrm{C}$ NMR $\delta(\square \mathrm{ppm} \square): 63.24\left(\mathrm{CH}_{2}\right), 110.14,116.26,116.28,116.38,118.13,119.72$, $121.57,121.79,122.61,122.81,122.83,123.75,123.77,129.25,129.38 ;$ HRMS: $m / z[\mathrm{M}+\mathrm{H}]^{+}$ calcd for $\mathrm{C}_{16} \mathrm{H}_{11} \mathrm{~N}_{2} \mathrm{OS}: 279.0592$; found 279.0603 .

6H-Pyrido[3", 2":2',3']pyrano[4',5':5,4] thiazolo[3,2-a]benzimidazole (4b). 49\% yield; mp $>230{ }^{\circ} \mathrm{C}$; LC-MS, $280\left(\mathrm{M}^{+}+1\right)$; ${ }^{1} \mathrm{H}$ NMR $\delta(\square \mathrm{ppm} \square)$ : 5.85 (s, 2H, 6-H), 7.03 (m, 1H, 3-H), 7.15 
(d, 1H, $J=8.4 \mathrm{~Hz}, 4-\mathrm{H}$ ), 7.24 and 7.34 (both t, 2H, $J=8.0 \mathrm{~Hz}, 8-\mathrm{H}$ and 9-H), 7.47 and 7.76 (both d, 2H, $J=8.0 \mathrm{~Hz}, 7-\mathrm{H}$ and $10 \mathrm{H}), 9.09$ (d, $1 \mathrm{H}, J=4.8 \mathrm{~Hz}, 2-\mathrm{H}) ;{ }^{13} \mathrm{C} \mathrm{NMR} \delta(\square \operatorname{ppm} \square)$ : $63.87\left(\mathrm{CH}_{2}\right), 110.08,117.63,119.75,121.69,122.26,123.47,124.04,124.84,129.17,119.97$, 137.87, 143.00, 147.99, 156.76; HRMS: $m / z[\mathrm{M}+\mathrm{H}]^{+}$calcd for $\mathrm{C}_{15} \mathrm{H}_{10} \mathrm{~N}_{2} \mathrm{OS}$ : 280.0545; found 280.0531 .

6H-[1]Benzopyrano[3',4':4,5]imidazo[2,1-b]benzothiazole (7). 63\% yield; $\mathrm{mp} 153-155{ }^{\circ} \mathrm{C}$; LC-MS, $279\left(\mathrm{M}^{+}+1\right)$; ${ }^{1} \mathrm{H}$ NMR $\delta(\square \mathrm{ppm} \square)$ : 5.29 (s, 2H, 6-H), 7.07-7.21 and 7.26-7.49 (m, 5H, 2-H, 3-H, 4-H, 10-H and 11-H), $7.72(\mathrm{~m}, 2 \mathrm{H}, 1-\mathrm{H}, 12-\mathrm{H}), 8.14(\mathrm{t}, 1 \mathrm{H}, J=8.4 \mathrm{~Hz}, 9-\mathrm{H}) ;{ }^{13} \mathrm{C}$ NMR $\delta(\square \operatorname{ppm} \square): 60.36\left(\mathrm{CH}_{2}\right), 114.16,114.80,117.98,118.09,121.80,122.29,124.49,124.79$, 125.93, 127.61, 130.00, 132.79, 142.08, 149.43, 152.51; HRMS: $m / z[\mathrm{M}+\mathrm{H}]^{+}$calcd for $\mathrm{C}_{16} \mathrm{H}_{11} \mathrm{~N}_{2} \mathrm{OS}$ : 279.0592; found 279.0584.

6H-[1]Benzopyrano[3',4':4,5]thiazolo[3,2-b][1,2,4]triazole (10). 31\% yield; mp 131-132 ${ }^{\circ} \mathrm{C}$; LC-MS, $230\left(\mathrm{M}^{+}+1\right)$; ${ }^{1} \mathrm{H}$ NMR $\delta(\square \mathrm{ppm} \square)$ : $\square 5.58$ (s, 2H, 6-H), 6.97 (d, 1H, J = 8.4 Hz, 4-H), $7.01(\mathrm{t}, 1 \mathrm{H}, J=7.6 \mathrm{~Hz}, 2-\mathrm{H}), 7.16(\mathrm{~d}, 1 \mathrm{H}, J=8.0 \mathrm{~Hz}, 1-\mathrm{H}), 7.22(\mathrm{t}, 1 \mathrm{H}, J=8.0 \mathrm{~Hz}, 3-\mathrm{H}), 8.13$ (s, $1 \mathrm{H}, 8-\mathrm{H}) ;{ }^{13} \mathrm{C}$ NMR $\delta(\square \mathrm{ppm} \square): 62.73\left(\mathrm{CH}_{2}\right), 116.75,117.54,120.59,121.53,122.58,122.81$, 130.07, 151.90, 155.87, 156.18; HRMS: $m / z[\mathrm{M}+\mathrm{H}]^{+}$calcd for $\mathrm{C}_{11} \mathrm{H}_{8} \mathrm{~N}_{3} \mathrm{OS}: 230.0388$; found 230.0399 .

9-Methylsulfanyl-6H-[1]Benzopyrano[3', $\left.\mathbf{4}^{\prime}: 4,5\right]$ imidazo[2,1-b][1,3,4]thiadiazole (13). $47 \%$ yield; mp 153-155 ${ }^{\circ} \mathrm{C}$; LC-MS, $276\left(\mathrm{M}^{+}+1\right)$; ${ }^{1} \mathrm{H}$ NMR $\delta$ ( $\square$ ppm $\square$ ): 2.82 (s, 3H, $\mathrm{CH}_{3}$ ), 5.47 (s, $2 \mathrm{H}, \mathrm{CH}_{2}$ ), $6.93(\mathrm{~d}, 1 \mathrm{H}, J=8.0 \mathrm{~Hz}, 4-\mathrm{H}$ ), 7.00 and 7.73 (both t, $2 \mathrm{H}, J=7.6 \mathrm{~Hz}, 2-\mathrm{H}$ and $3-\mathrm{H}$ ), $7.73(\mathrm{~d}, 1 \mathrm{H}, J=7.6 \mathrm{~Hz}, 1-\mathrm{H}) ;{ }^{13} \mathrm{C}$ NMR $\delta(\square \mathrm{ppm} \square)$ : $16.24\left(\mathrm{CH}_{3}\right), 67.72\left(\mathrm{CH}_{2}\right), 115.70,116.50$, 120.73, 121.64, 121.65, 128.11, 136.09, 145.30, 151.56, 161.82; HRMS: $m / z[\mathrm{M}+\mathrm{H}]^{+}$calcd for $\mathrm{C}_{12} \mathrm{H}_{10} \mathrm{~N}_{3} \mathrm{OS}_{2}$ : 276.0265; found 276.0256.

In vitro cytotoxicity assay. Monolayer tumor cell lines -HT-1080 (human fibrosarcoma), MG-22A (mouse hepatoma), 3T3 (mouse Swiss Albino embryo fibroblasts), - were cultured in standard medium (Dulbecco`s modified Eagle`s medium; DMEM) and supplemented with 10\% fetal bovine serum ("Sigma"). Tumor cell lines were obtained from the ATCC. About $10 \times 10^{4}$ cells $\mathrm{ml}^{-1}$ were placed in 96-well plates immediately after compounds were added to the wells; the volume of each plate was $200 \mu$. The control cells without test compounds were cultured on separate plate. The plates were incubated for $72 \mathrm{~h}, 37^{\circ} \mathrm{C}, 5 \% \mathrm{CO}_{2}$. The number of surviving cells was determined using tri(4-dimethylaminophenyl)methyl chloride (crystal violet: CV) or 3-(4,5dimethylthiazol-2-yl)-2,5-diphenyltetrazolinium bromide (MTT) ${ }^{30,31}$. LD $_{50}$ was tested according "Alternative Toxicological Methods". ${ }^{32}$ The program Graph Pad Prism ${ }^{\circledR} 3.0$ was used for calculations ( $\mathrm{r} \square<0.05$.).

\section{Acknowledgements}


This work was supported by the project of ESF Foundation of Latvia (Project No. 2009/0197/1DP/1.1.1.2.0/09/APIA/VIAA/014).

\section{References}

1. Rocha, D. F. O.; Hamilton, K.; Goncalves, C. C. S.; Machado, G.; Marsaioli, A. J. J. Nat. Prod. 2011, 74, 658.

2. Mulwad, V. V.; Langi, B. P.; Chaskar, A. C. Acta. Poloniae Pharm.-Drug Res. 2011, 68, 39.

3. Agata, N.; Nogi, H.; Milhollen, M.; Kharbanda, S.; Kufe, D. Cancer Res. 2004, 64, 8512.

4. Ghosh, C. K.; Patra, A. J. Heterocycl. Chem. 2008, 45, 1529.

5. Ghosh, C. K. J. Heterocycl. Chem. 2006, 43, 813.

6. Katritzky, A. R.; Ramsden, C. A.; Scriven, E. F. V.; Taylor, R. J. K. Six-membered rings with one heteroatom, and their fused carbocyclic derivatives. Comprehensive Heterocyclic Chemistry III, 2008, 7, 1-1066.

7. Hepworth, J. D.; Heron, B. M. Progr. Heterocycl. Chem. 2009, 21, 455.

8. Goel, A.; Ram, V. J. Tetrahedron 2009, 65, 7865.

9. Ram, V. J.; Srivastava, P. Curr. Org. Chem. 2001, 5, 571.

10. Sorkin, E.; Krähenbühl, W.; Erlenmeyer, H. Helv. Chim. Acta 1948, 31, 65.

11. Colotta, V.; Catarzi, D.; Varano, F.; Cecchi, L.; Filacchioni, G.; Martini, C.; Giusti, L.; Lucacchini, A. Farmaco 1998, 53, 375.

12. Che, Q.; Vo, N. H.; Chen, S. US Patent 0132513A1, 2008.

13. Potts, K. T.; Dery, M. O. J. Chem. Soc., Chem. Commun. 1986, 561.

14. Bellec, C.; Vinot, N.; Maitte, P. J. Heterocycl. Chem. 1986, 23, 491.

15. Chiodini, L.; Di Ciommo, M.; Merlini, L. J. Heterocycl. Chem. 1981, 18, 23.

16. Seregin, I. V.; Gevorgyan, V. Chem. Soc. Rev. 2007, 36, 1173.

17. Majumdar, K.; Chattopadhyay, B. Curr. Org. Chem. 2009, 13, 731.

18. Heravi, M. M.; Fazeli, A. Heterocycles 2010, 81, 1979.

19. Majumdar, K. C.; Chattopadhyay, B.; Maji, P. K.; Chattopadhyay, S. K.; Samanta, S. Heterocycles 2010, 81, 517.

20. Bellina, F.; Rossi, R. Adv. Synth. Catal. 2010, 352, 1223.

21. Bahlaouan, Z.; Abarbri, M.; Duchene, A.; Thibonnet, J.; Henry, N.; Enguehard-Gueiffier, C.; Gueiffier, A. Org. Biomol. Chem. 2011, 9, 1212.

22. Beresņeva, T.; Ābele, E.; Šestakova, I.; Jaschenko, E.; Bridane, V.; Kalviņš, I. Latv. J. Chem. 2009, 133.

23. Chimirri, A.; Grasso, S.; Romeo, G.; Zappala, M. Heterocycles 1988, 27, 1975.

24. Beresneva, T.; Abele, E. Heterocyclic Lett. 2011, 1, 73.

25. Yanagisawa, S.; Sudo, T.; Noyori, R.; Itami, K. J. Am. Chem. Soc. 2006, 128, 11748.

26. Van der Helm, D.; Powell, D. R.; Berlin, K. D.; Spruce, L. W.; Shyamasundar, N.; Radhakrishna, A. S. Acta Cryst. 1987, C43, 1723. 
27. Anilkumar, G. N.; Kokila, M. K.; Puttaraja; Karki, S. S.; Kulkarni, M. V. Acta Cryst. 2006, E62, 2014.

28. Burla, M. C.; Caliandro, R.; Camalli, M.; Carrozzini, B.; Cascarano, G. L.; De Caro, L.; Giacovazzo, C.; Polidori, G.; Spagna, R. J. Appl. Cryst. 2005, 38, 381.

29. Sheldrick, G.M. SHELXL97. University of Göttingen, Germany, 1997.

30. Fast, D. J.; Lynch, R. C.; Leu, R. W. J. Leuckocyt. Biol. 1992, $52,255$.

31. Freshney, P. J. Culture of Animal Cells (A Manual of Basic Technique), Wiley-Liss, New York, 1994, pp. 296-297.

32. http://iccvam.niehs.nih.gov/methods/invidocs/guidance/iv_guide.htm [2004.01.10]. 